Article

\title{
Logarithm of a Non-Singular Complex Matrix via the Dunford-Taylor Integral
}

\author{
Diego Caratelli ${ }^{1,2}$ (D) and Paolo Emilio Ricci ${ }^{3, *}$ (1) \\ 1 Department of Research and Development, The Antenna Company, High Tech Campus 29, \\ 5656 AE Eindhoven, The Netherlands; diego.caratelli@antennacompany.com or d.caratelli@tue.nl \\ 2 Electromagnetics Group, Department of Electrical Engineering, Eindhoven University of Technology, \\ P.O. Box 513, $5600 \mathrm{MB}$ Eindhoven, The Netherlands \\ 3 Dipartimento di Matematica, International Telematic University UniNettuno, 39 Corso Vittorio Emanuele II, \\ I-00186 Rome, Italy \\ * Correspondence: p.ricci@uninettunouniversity.net or paoloemilioricci@gmail.com
}

check for updates

Citation: Caratelli, D.; Ricci, P.E. Logarithm of a Non-Singular Complex Matrix via the Dunford-Taylor Integral. Axioms 2022, 11, 51. https://doi.org/ 10.3390/axioms11020051

Academic Editor: Hari Mohan Srivastava

Received: 22 December 2021

Accepted: 25 January 2022

Published: 27 January 2022

Publisher's Note: MDPI stays neutral with regard to jurisdictional claims in published maps and institutional affiliations.

Copyright: (c) 2022 by the authors. Licensee MDPI, Basel, Switzerland. This article is an open access article distributed under the terms and conditions of the Creative Commons Attribution (CC BY) license (https:// creativecommons.org/licenses/by/ $4.0 /)$.

\begin{abstract}
Using the Dunford-Taylor integral and a representation formula for the resolvent of a non-singular complex matrix, we find the logarithm of a non-singular complex matrix applying the Cauchy's residue theorem if the matrix eigenvalues are known or a circuit integral extended to a curve surrounding the spectrum. The logarithm function that can be found using this technique is essentially unique. To define a version of the logarithm with multiple values analogous to the one existing in the case of complex variables, we introduce a definition for the argument of a matrix, showing the possibility of finding equations similar to those of the scalar case. In the last section, numerical experiments performed by the first author, using the computer algebra program Mathematica $($, confirm the effectiveness of this methodology. They include the logarithm of matrices of the fifth, sixth and seventh order.
\end{abstract}

Keywords: Dunford-Taylor integral; matrix functions; matrix logarithm

MSC: primary 15A60; secondary 47A10; 65F60

\section{Introduction}

Matrix functions are an important topic [1,2] due to their applications in many branches of applied mathematics, physics, statistics and engineering. In particular, the matrix exponential and its inverse, the matrix logarithm, appear in so many research fields that it would be almost impossible to collect all citations.

Computation methods for the principal value of the matrix logarithm can be found in several articles using different approaches. In [3], an improvement of the inverse scaling and squaring method is shown; in [4], an alternative formulation of the matrix arithmeticgeometric mean algorithm is used; in [5], multiprecision algorithms are analyzed; in [6], algorithms based on a numerical spectral decomposition or on a numerical Jordan decomposition are used; in [7], algorithms based on the double exponential formula are used; and in [8], an improved Taylor algorithm is applied.

However, the closest example to our approach is the paper by N. Hale, N.J. Higham, N.J. and L.N. Trefethen [9], using functional analysis and contour integrals.

This approach was recently used by the authors in order to find the most common matrix functions, as the matrix roots [10], the inverse of a matrix and in particular the inverse of a tridiagonal matrix [11]. Previous articles were dedicated to the matrix exponential [12-14], recalling that its definition by MacLaurin expansion is useless, since according to F.R. Gantmacher [1] the exponential of a matrix $\mathcal{A}$ is just the matrix polynomial interpolating the exponential function on the spectrum of $\mathcal{A}$. 
In our opinion, this article goes beyond the results obtained in [9] since in that article the representation formula of the resolvent in terms of the matrix invariants is missing. This equation can be found in an old book by S. Cherubino [15]. Since this book is written in Italian, his knowledge probably escaped those authors.

Therefore, we are able to represent the logarithm of a non-singular complex matrix using the Dunford-Taylor integral and then apply Cauchy's residue theorem if the matrix eigenvalues are known, or a simple circuit integral on a regular curve surrounding the spectrum, avoiding this knowledge.

This can be done since it is always possible to find a ray $r$ coming out from the origin not intersecting the spectrum of $\mathcal{A}$ and therefore to consider a regular curve $\gamma$ surrounding all the eigenvalues and such that $r \cap \gamma=\varnothing$.

Of course, the obtained value of $\log \mathcal{A}$ is independent of the used ray $r$, so that in order to introduce a multiple-valued logarithm, as in the case of complex numbers, it is necessary to define the argument of a matrix.

This was done in Section 4.1, introducing two possible definitions for the argument of a matrix, depending on whether one assumes the scalar or matrix definition for the argument (the second definition holding in the space of commuting matrices). Then, several branches of the logarithm function can be considered, in a similar way to the complex variable function.

Of course, only the principal branch is often requested, and this is obtained in a trivial way provided that the matrix does not have real negative eigenvalues.

In the last section, some numerical experiments were made by the first author using the computer algebra program Mathematica $($, confirming the effectiveness of the proposed method.

\section{Basic Definitions}

Let $\mathcal{A}=\left(a_{i j}\right)$, be an $r \times r$, non-singular complex matrix. Its invariants, so-called because of being invariant under similarity transformations [16], are given by

$$
u_{1}=\operatorname{tr} \mathcal{A}, \quad u_{2}=\sum_{i<j}^{1, r}\left|\begin{array}{ll}
a_{i i} & a_{i j} \\
a_{j i} & a_{j j}
\end{array}\right|, \quad \cdots, \quad u_{r}=\operatorname{det} \mathcal{A},
$$

Using for brevity $\mathbf{u}=\left(u_{1}, u_{2}, \ldots, u_{r}\right)$ and $\left(u_{0}=1\right)$, its characteristic polynomial can be written as

$$
P(\mathbf{u} ; \lambda)=\operatorname{det}(\lambda \mathcal{I}-\mathcal{A})=\lambda^{r}-u_{1} \lambda^{r-1}+u_{2} \lambda^{r-2}+\ldots+(-1)^{r} u_{r}
$$

and the relevant characteristic equation is

$$
P(\lambda)=P(\mathbf{u} ; \lambda)=0
$$

The roots of $P(\lambda)$,

$$
\lambda_{1}, \lambda_{2}, \ldots, \lambda_{r}
$$

are the eigenvalues of $\mathcal{A}$.

S. Cherubino (pp. 93-95 [15]) proved that the resolvent of $\mathcal{A}$, in terms of the invariants, is represented by the equation

$$
(\lambda \mathcal{I}-\mathcal{A})^{-1}=\frac{1}{P(\lambda)} \sum_{k=0}^{r-1}\left[\sum_{h=0}^{r-k-1}(-1)^{h} u_{h} \lambda^{r-k-h-1}\right] \mathcal{A}^{k}
$$

\section{The Dunford-Taylor Integral}

The Dunford-Taylor integral $[17,18]$ is a classical tool in functional analysis. It is also ascribed to Frigyes Riesz [19] and Luigi Fantappiè [20]. 
This integral, for holomorphic functions of an operator, takes the place of Cauchy's integral in function theory. In the finite dimensional case, the matrix $\mathcal{A}$ is the operator under consideration. The result is as follows:

Theorem 1. Suppose that $f(\lambda)$ is a holomorphic function in a domain $\Delta \subset \mathbf{C}$, containing all the eigenvalues $\lambda_{h}$ of $\mathcal{A}$, and let $\gamma \subset \Delta$ be a closed piece-wise smooth curve with positive direction encircling all the eigenvalues.

Then, the matrix function $f(\mathcal{A})$ is defined by the Dunford-Taylor integral:

$$
f(\mathcal{A})=\frac{1}{2 \pi \mathrm{i}} \oint_{\gamma} f(\lambda)(\lambda \mathcal{I}-\mathcal{A})^{-1} d \lambda
$$

where $(\lambda \mathcal{I}-\mathcal{A})^{-1}$ denotes the resolvent of $\mathcal{A}$.

The application of the Dunford-Taylor integral requires only the knowledge of matrix invariants, which are derived from matrix entries. Instead, to apply Cauchy's residue theorem, it is mandatory to know the eigenvalues and their multiplicity. Therefore, the first technique is sometimes computationally more convenient.

In [10], we have used the Dunford-Taylor integral in order to construct the roots of a non-singular complex matrix $\mathcal{A}$ according to the equation:

$$
\mathcal{A}^{1 / n}=\frac{1}{2 \pi \mathrm{i}} \oint_{\gamma} \lambda^{1 / n}(\lambda \mathcal{I}-\mathcal{A})^{-1} d \lambda
$$

By using Equation (5), Theorem 1 becomes

Theorem 2. Let $f(\lambda)$ be a holomorphic function in a domain $\Delta \subset \mathbf{C}$, containing the spectrum of $\mathcal{A}$, and denote by $\gamma \subset \Delta$ a piece-wise smooth contour encircling the spectrum. Then, Equation (6) is written as

$$
f(\mathcal{A})=\frac{1}{2 \pi \mathrm{i}}\left[\sum_{k=1}^{r} \oint_{\gamma} \frac{f(\lambda) \sum_{h=0}^{k-1}(-1)^{h} u_{h} \lambda^{k-h-1}}{P(\lambda)} d \lambda \mathcal{A}^{r-k}\right] .
$$

Remark 1. From Cauchy's bounds for the roots of polynomials [21,22], it immediately follows that, in case of the characteristic Equation (5), the highest (in modulus) eigenvalue is bounded by

$$
1+\max \left\{\frac{\left|u_{r-1}\right|}{\left|u_{r}\right|}, \frac{\left|u_{r-2}\right|}{\left|u_{r}\right|}, \cdots, \frac{1}{\left|u_{r}\right|}\right\} .
$$

Making the substitution $\mu=\lambda^{-1}$ in the polynomial (4), multiplying by $\mu^{r}$ and applying the Cauchy bound, we find the upper bound of the zeros of $\mu^{r} P\left(\mu^{-1}\right)$. Then, returning to the variable $\lambda$, we find the lower bound to the roots of $P(\lambda)$, which is given by

$$
\left[1+\max \left\{\left|u_{1}\right|,\left|u_{2}\right|, \ldots,\left|u_{r}\right|\right\}\right]^{-1} \text {. }
$$

These bounds can be used in order to construct the curve $\gamma$ in Theorem 2.

\subsection{The Case of Logarithm}

Looking for the logarithm of a matrix $\mathcal{A}$, it is necessary for us to recall that the complex function $\log z$ is not holomorphic in the whole plane, but in an open set obtained by cutting the plane along a ray coming out from the origin. The inclination of this ray determines the branch of the logarithm. Cutting off the negative semi-axis we have the principal logarithm.

From the point of view of functional analysis, it can be said that the logarithm of a matrix only depends on the set of its eigenvalues. It is mandatory to assume that the matrix is invertible, meaning that the zero is not an eigenvalue. Assuming this condition, for any 
ray coming out from the origin which does not intersect the spectrum of $\mathcal{A}$, it is possible to define the matrix $\mathcal{X}=\log \mathcal{A}$, which satisfies the defining condition

$$
e^{\mathcal{X}}=\mathcal{A}
$$

It is possible to define the principal value of $\log \mathcal{A}$ if and only if the matrix $\mathcal{A}$ does not have real negative eigenvalues; in particular, if the matrix is positive definite. In this case, a possible path for the $\gamma$ curve, encircling all eigenvalues, is given in Figure 1.

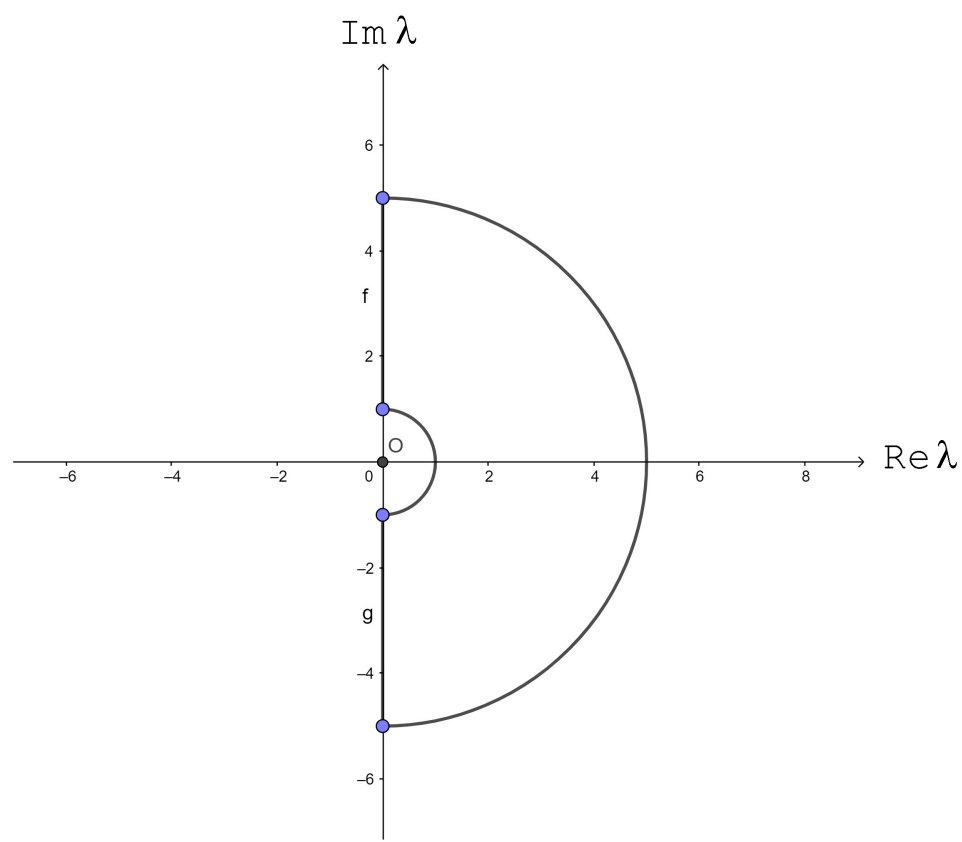

Figure 1. Path of $\gamma$ curve enclosing all eigenvalues for a positive definite matrix.

Supposing the matrix $\mathcal{A}$, is stable, so that there do not exist eigenvalues with a positive real part, and assuming that all eigenvalues are complex, it is possible to define the matrix $\log \mathcal{A}$, using a curve $\gamma$ similar to that depicted in Figure 2.

Remark 2. Apparently, once we have defined the ray coming out from the origin which does not intersect the spectrum of $\mathcal{A}$, the numerical entries of the corresponding matrix $\log A$ are independent of the considered ray, as Cauchy's residue theorem or the Dunford-Taylor integral always give the same result. Therefore, from the point of view of functional analysis, the log function is unique.

In our opinion, in order to overcome this problem, it is necessary to define the argument of a matrix. 


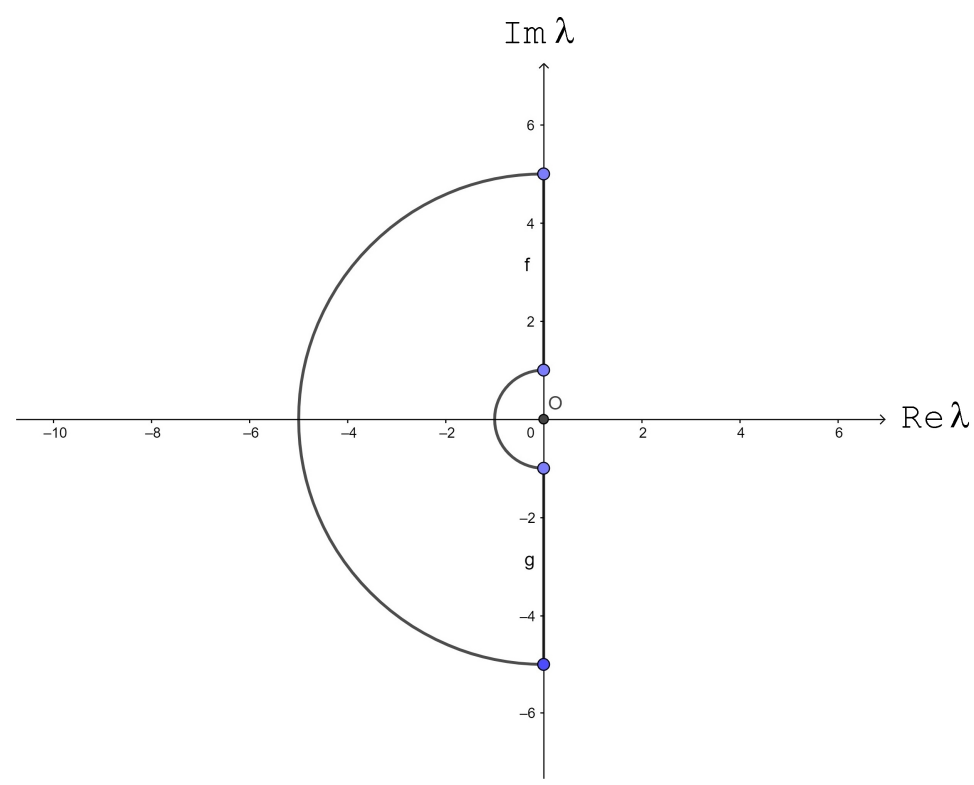

Figure 2. Path of $\gamma$ curve enclosing all eigenvalues for a stable matrix.

\subsection{The Argument Definitions}

Depending on whether the scalar or matrix definition for the argument is assumed, we can introduce the two following definitions, with the second one holding only for commuting matrices.

Definition 1. We define the scalar argument of a non-singular complex matrix $\mathcal{A}$ as the argument $\theta$ of the determinant of $\mathcal{A}$ :

$$
\arg \mathcal{A}=\arg (\operatorname{det} \mathcal{A})=\theta .
$$

Then, we can write the matrix $\mathcal{A}$, taking into account its argument, in the form

$$
\mathcal{Z}=\mathcal{A} e^{i \theta}
$$

Considering another matrix $\mathcal{B}$, whose argument is denoted by $\phi=\arg (\operatorname{det} \mathcal{B})$, we find

$$
\mathcal{A} e^{i \theta} \mathcal{B} e^{i \phi}=\mathcal{A B} e^{i(\theta+\phi)},
$$

and the argument of $\mathcal{A B}$ is $\theta+\phi$, since

$$
\arg (\mathcal{A B})=\arg [\operatorname{det}(\mathcal{A B})]=\arg (\operatorname{det} \mathcal{A} \operatorname{det} \mathcal{B})=\arg (\operatorname{det} \mathcal{A})+\arg (\operatorname{det} \mathcal{B}) \text {. }
$$

Note that, using this definition, the only matrices whose argument is indeterminate are the singular matrices, which are excluded in our approach.

Choosing a ray coming out from the origin and not intersecting the spectrum of $\mathcal{A}$, consider the logarithm of $\mathcal{A}$, according to Theorem 2.

Then, denoting by $\mathcal{I}$ the $r \times r$ unit matrix, since the diagonal matrix $i(\theta+2 k \pi) \mathcal{I}$ commutes with $\log (\mathcal{A})$, we find

$$
\mathcal{Z}=e^{\mathcal{X}} \quad \text { iff } \quad \mathcal{X}=\log (\mathcal{A})+i(\theta+2 k \pi) \mathcal{I}, \quad(k \in \mathbf{Z}) .
$$

Definition 2. Considering the space of non-singular commuting matrices, we define the matrix modulus as $|\mathcal{A}|=\exp [\Re(\log (\mathcal{A}))]$ and the matrix argument as $\arg (\mathcal{A})=\Im(\log (\mathcal{A}))$. Even in this case, we find

$$
\arg (\mathcal{A B})=\arg (\mathcal{A})+\arg (\mathcal{B})
$$


Denoting by $\mathcal{U}$ the $r \times r$ matrix $\mathcal{U}=\left\{a_{h, k} \equiv 1\right\}_{\forall h, k=1,2, \ldots, r^{\prime}}$ since the matrix $i(\theta+2 k \pi) \mathcal{U}$ commutes with $\log (\mathcal{A})$, we find again that

$$
\mathcal{Z}=e^{\mathcal{X}} \quad \text { iff } \quad \mathcal{X}=\log (\mathcal{A})+i(\theta+2 k \pi) \mathcal{U}, \quad(k \in \mathbf{Z}) .
$$

This gives the possibility of distinguishing the different branches of $\log (\mathcal{A})$, since the $\log$ function becomes multiple-valued.

\section{Numerical Experiments}

4.1. Logarithm of a Non-Singular Matrix of Fifth Order

Let us consider the real-valued matrix:

$$
\mathcal{A}=\left(\begin{array}{ccccc}
3 & -2 & -4 & -1 & 2 \\
2 & 3 & -2 & -4 & -1 \\
3 & 0 & 4 & 2 & -1 \\
4 & 4 & 1 & -1 & -1 \\
0 & 3 & 0 & -2 & 3
\end{array}\right)
$$

The relevant invariants are

$$
u_{1}=12, u_{2}=85, \quad u_{3}=345, \quad u_{4}=922, u_{5}=976 .
$$

Therefore, it is not difficult to evaluate the matrix eigenvalues as follows:

$$
\begin{aligned}
& \lambda_{1} \simeq 3.57665+\mathrm{i} 3.36026, \\
& \lambda_{2} \simeq 3.57665-\mathrm{i} 3.36026, \\
& \lambda_{3} \simeq 1.43877+\mathrm{i} 4.30232, \\
& \lambda_{4} \simeq 1.43877-\mathrm{i} 4.30232, \\
& \lambda_{5} \simeq 1.96915 .
\end{aligned}
$$

By using the Dunford-Taylor integral Formula (8) in combination with the Gauss-Kronrod

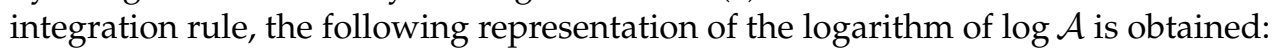

$$
\log \mathcal{A}=\sum_{k=1}^{r} \xi_{k} \mathcal{A}^{r-k}
$$

with

$$
\begin{aligned}
& \xi_{1} \simeq-0.00128030, \\
& \xi_{2} \simeq 0.0173067, \\
& \xi_{3} \simeq-0.140009, \\
& \xi_{4} \simeq 0.784704, \\
& \xi_{5} \simeq-0.437600,
\end{aligned}
$$

leading to the conclusion that

$$
\log \mathcal{A} \simeq\left(\begin{array}{ccccc}
1.66590 & -0.355067 & -0.825128 & -0.285264 & 0.260402 \\
0.732525 & 2.36326 & 0.372011 & -1.42119 & -0.393748 \\
0.466116 & -0.306649 & 1.34268 & 0.86526 & -0.342701 \\
0.608954 & 1.85113 & 1.07661 & 0.221497 & -0.0587207 \\
-0.194051 & 0.829696 & 0.0637407 & -0.217737 & 1.29012
\end{array}\right)
$$

By using (13), it can be readily verified that

$$
e^{\log \mathcal{A}}-\mathcal{A} \simeq \mathcal{O}_{5},
$$

with $\mathcal{O}_{5}$ denoting the zero matrix of order 5 . 
4.2. Logarithm of a Non-Singular Matrix of Sixth Order

Let us consider the complex-valued matrix:

$$
\mathcal{A}=\left(\begin{array}{cccccc}
4+\mathrm{i} & 5+2 \mathrm{i} & -5-5 \mathrm{i} & -3-4 \mathrm{i} & -5-\mathrm{i} & -1-\mathrm{i} \\
-5-3 \mathrm{i} & 3-2 \mathrm{i} & 4+2 \mathrm{i} & 3-2 \mathrm{i} & 0 & 2+2 \mathrm{i} \\
2-5 \mathrm{i} & 4 & 3-5 \mathrm{i} & -3-2 \mathrm{i} & 2-5 \mathrm{i} & 3+2 \mathrm{i} \\
5+4 \mathrm{i} & -3+2 \mathrm{i} & 4-3 \mathrm{i} & 5+\mathrm{i} & -3+2 \mathrm{i} & -4+5 \mathrm{i} \\
4-3 \mathrm{i} & 5+4 \mathrm{i} & -3-3 \mathrm{i} & 2+5 \mathrm{i} & 2-4 \mathrm{i} & -1-4 \mathrm{i} \\
2-4 \mathrm{i} & 1-2 \mathrm{i} & 5 & 4+2 \mathrm{i} & -2 \mathrm{i} & 5+5 \mathrm{i}
\end{array}\right)
$$

The relevant invariants are

$$
\begin{aligned}
& u_{1}=22-4 \mathrm{i}, \\
& u_{2}=365-105 \mathrm{i}, \\
& u_{3}=4218-1068 \mathrm{i}, \\
& u_{4}=29,944-6663 \mathrm{i}, \\
& u_{5}=138,548-14,107 \mathrm{i}, \\
& u_{6}=295,151+54,602 \mathrm{i} .
\end{aligned}
$$

Therefore, it is not difficult to evaluate the matrix eigenvalues as follows:

$$
\begin{aligned}
& \lambda_{1} \simeq 0.296978-\mathrm{i} 14.4801, \\
& \lambda_{2} \simeq 1.78713+\mathrm{i} 10.3038, \\
& \lambda_{3} \simeq 8.46023+\mathrm{i} 0.493258, \\
& \lambda_{4} \simeq 3.35404-\mathrm{i} 6.71629, \\
& \lambda_{5} \simeq 5.64303+\mathrm{i} 1.72914, \\
& \lambda_{6} \simeq 2.45859+\mathrm{i} 4.67021 .
\end{aligned}
$$

By using the Dunford-Taylor integral Formula (8) in combination with the Gauss-Kronrod integration rule, the following representation of the logarithm of $\log \mathcal{A}$ is obtained:

$$
\log \mathcal{A}=\sum_{k=1}^{r} \xi_{k} \mathcal{A}^{r-k}
$$

with

$$
\begin{aligned}
& \xi_{1} \simeq 0.00000770000 \\
& \xi_{2} \simeq-0.0001897+\mathrm{i} 0.0000290000 \\
& \xi_{3} \simeq 0.00338310-\mathrm{i} 0.000829900 \\
& \xi_{4} \simeq-0.0441450+\mathrm{i} 0.00946410 \\
& \xi_{5} \simeq 0.432130-\mathrm{i} 0.0667950 \\
& \xi_{6} \simeq 0.223944+\mathrm{i} 0.226711
\end{aligned}
$$

leading to the conclusion that

$$
\log \mathcal{A}=\operatorname{Re}\{\log \mathcal{A}\}+\mathrm{i} \operatorname{Im}\{\log \mathcal{A}\}
$$

with

$$
\simeq\left(\begin{array}{cccccc}
2.35243 & \operatorname{Re}\{\log \mathcal{A}\} \simeq \\
-0.909448 & 1.62347 & 0.391308 & 0.242446 & -0.416987 & 0.130259 \\
0.153646 & -0.163990 & 2.14103 & -0.511755 & 0.416629 & 0.263935 \\
0.408941 & -0.341896 & 0.707356 & 2.47080 & -0.535530 & -0.434344 \\
0.947460 & 0.385044 & -0.110443 & 0.212405 & 2.07195 & 0.195797 \\
-0.184988 & -0.0292639 & 0.807245 & 0.761308 & 0.0799232 & 1.95240
\end{array}\right),
$$




$$
\simeq\left(\begin{array}{cccccc}
0.489305 & 0.437798 & -0.980096 & -0.388717 & -0.0228187 & 0.153350 \\
-0.135544 & 0.0801520 & 0.104508 & -0.293374 & -0.253805 & -0.183412 \\
0.00491990 & -0.0106664 & -0.706470 & -0.642022 & -0.536870 & 0.495318 \\
0.114840 & 0.183967 & -0.249145 & 0.233061 & 0.474526 & 0.564281 \\
-0.126378 & 1.00845 & -0.488570 & 0.772015 & -0.220680 & -0.530668 \\
-0.505809 & -0.0749493 & 0.0377549 & 0.157431 & -0.371643 & 0.307561
\end{array}\right)
$$

By using (15), it can be readily verified that

$$
e^{\log \mathcal{A}}-\mathcal{A} \simeq \mathcal{O}_{6},
$$

with $\mathcal{O}_{6}$ denoting the zero matrix of order 6 .

4.3. Logarithm of a Non-Singular Matrix of Seventh Order

Let us consider the complex-valued matrix:

$$
\mathcal{A}=\left(\begin{array}{ccccccc}
6+6 \mathrm{i} & -4-3 \mathrm{i} & 6+3 \mathrm{i} & 1+4 \mathrm{i} & 4+2 \mathrm{i} & -4+5 \mathrm{i} & -4+6 \mathrm{i} \\
4-\mathrm{i} & 5-4 \mathrm{i} & -5+3 \mathrm{i} & -5-2 \mathrm{i} & -5-2 \mathrm{i} & 1+6 \mathrm{i} & -5-2 \mathrm{i} \\
-6+3 \mathrm{i} & -3+4 \mathrm{i} & 6-3 \mathrm{i} & -2-5 \mathrm{i} & 3-4 \mathrm{i} & -6-5 \mathrm{i} & -4-6 \mathrm{i} \\
5 \mathrm{i} & 2-5 \mathrm{i} & 5-6 \mathrm{i} & -2+4 \mathrm{i} & -3-2 \mathrm{i} & 4-4 \mathrm{i} & -\mathrm{i} \\
-3-\mathrm{i} & 4+4 \mathrm{i} & 2-6 \mathrm{i} & 4-\mathrm{i} & 4-6 \mathrm{i} & 1+\mathrm{i} & 3-2 \mathrm{i} \\
6+4 \mathrm{i} & -1-2 \mathrm{i} & 4+4 \mathrm{i} & -4+3 \mathrm{i} & 1-3 \mathrm{i} & 6-3 \mathrm{i} & 3+2 \mathrm{i} \\
1+3 \mathrm{i} & 6-3 \mathrm{i} & 5 & -4 & -3 & 6+6 \mathrm{i} & 5+5 \mathrm{i}
\end{array}\right)
$$

The relevant invariants are

$$
\begin{aligned}
& u_{1}=30-\mathrm{i}, \\
& u_{2}=747+95 \mathrm{i}, \\
& u_{3}=10,340+251 \mathrm{i}, \\
& u_{4}=88,078+4041 \mathrm{i}, \\
& u_{5}=595,672-133,464 \mathrm{i} \\
& u_{6}=1,843,947-1,021,663 \mathrm{i}, \\
& u_{7}=1,506,786-1,823,100 \mathrm{i} .
\end{aligned}
$$

Therefore, it is not difficult to evaluate the matrix eigenvalues as follows:

$$
\begin{aligned}
& \lambda_{1} \simeq 2.95932+\mathrm{i} 19.2252, \\
& \lambda_{2} \simeq 8.04205-\mathrm{i} 16.1180, \\
& \lambda_{3} \simeq 11.2570-\mathrm{i} 6.51033, \\
& \lambda_{4} \simeq 2.18318+\mathrm{i} 9.92636, \\
& \lambda_{5} \simeq 0.000165700-\mathrm{i} 6.82015, \\
& \lambda_{6} \simeq 3.87679+\mathrm{i} 0.240170, \\
& \lambda_{7} \simeq 1.68145-\mathrm{i} 0.943299 .
\end{aligned}
$$

By using the Dunford-Taylor integral Formula (8) in combination with the Gauss-Kronrod integration rule, the following representation of the $\log$ arithm of $\log \mathcal{A}$ is obtained:

$$
\log \mathcal{A}=\sum_{k=1}^{r} \xi_{k} \mathcal{A}^{r-k},
$$


with

$$
\begin{aligned}
& \xi_{1} \simeq-0.000000500000-\mathrm{i} 0.000000300000 \\
& \xi_{2} \simeq 0.0000173000+\mathrm{i} 0.00000990000 \\
& \xi_{3} \simeq-0.000402100-\mathrm{i} 0.000313700 \\
& \xi_{4} \simeq 0.00639060+\mathrm{i} 0.00404060 \\
& \xi_{5} \simeq-0.0628271-\mathrm{i} 0.0373443 \\
& \xi_{6} \simeq 0.599498+\mathrm{i} 0.185374 \\
& \xi_{7} \simeq-0.315331-\mathrm{i} 0.346162
\end{aligned}
$$

leading to the conclusion that

$$
\log \mathcal{A}=\operatorname{Re}\{\log \mathcal{A}\}+\mathrm{i} \operatorname{Im}\{\log \mathcal{A}\} .
$$

with

$$
\simeq\left(\begin{array}{ccccccc}
2.91362 & -0.817604 & 0.221965 & -0.0730755 & 0.208167 & -0.520366 & -0.129561 \\
0.567363 & 1.95258 & -0.104721 & -0.813830 & -1.02429 & -0.144333 & -0.607049 \\
-0.342212 & -0.426421 & 2.61202 & -0.821639 & 0.491866 & -0.0772535 & -0.352127 \\
-0.179357 & 0.137764 & 0.758419 & 1.15381 & 0.391973 & 0.773287 & 0.661585 \\
-0.275540 & -0.148037 & -0.0796344 & 1.06787 & 1.41416 & -0.396807 & -0.0560561 \\
0.920576 & -0.359465 & 0.246612 & -1.30242 & 0.510602 & 2.45570 & 0.781888 \\
-0.230439 & 1.02208 & 0.455449 & 0.889910 & -0.324223 & 0.548304 & 2.17447
\end{array}\right),
$$

$$
\simeq\left(\begin{array}{ccccccc}
0.420685 & -0.611369 & -0.11909 & 0.80744 & -0.32481 & 0.372724 & 0.199991 \\
-0.514289 & -0.210821 & -0.195845 & 0.586501 & -1.11125 & 0.559848 & -0.778494 \\
0.49197 & 0.057024 & -0.301965 & -0.371166 & -0.605538 & -0.122561 & -0.356149 \\
0.66582 & -0.997078 & -1.13623 & -0.027842 & -0.193776 & -0.564589 & -0.500757 \\
-0.667146 & 0.837475 & -0.153151 & 0.778914 & -0.572962 & 0.0110945 & 0.0047255 \\
0.691494 & -0.384569 & -0.0625811 & 0.188682 & -0.812471 & -0.919479 & -0.12588 \\
-0.164098 & -0.212392 & -0.0985634 & -0.226954 & 0.740542 & 0.691923 & 0.732278
\end{array}\right) .
$$

By using (17), it can be readily verified that:

$$
e^{\log \mathcal{A}}-\mathcal{A} \simeq \mathcal{O}_{7},
$$

with $\mathcal{O}_{7}$ denoting the zero matrix of order 7.

\section{Conclusions}

In preceding articles [10,11], we have shown that the Dunford-Taylor integral allows us to find the main holomorphic matrix functions of a non-singular complex matrix $\mathcal{A}$ provided that its invariants are known. In this paper, we considered the logarithm of $\mathcal{A}$ noting that even though the logarithm is not holomorphic throughout the plane, it is always possible to cut the plane along a ray $r$ that exits the origin and does not intersect the spectrum of $\mathcal{A}$. Then, by fixing a piece-wise smooth closed curve surrounding all the eigenvalues of $\mathcal{A}$ and that does not intersect $r$, we used the Dunford-Taylor integral to calculate $\log \mathcal{A}$. This gives a single value for the logarithm, regardless of the considered ray. To find a multi-valued function for the logarithm, we have defined in two different ways the argument of a non-singular complex matrix $\mathcal{A}$, depending on whether the scalar or the matrix definition for the argument is assumed (the second definition holding in the space of commuting matrices). This gives the different branches of the logarithm in a way similar to that existing for the theory of complex variables. The numerical experiments, contained in the last section and applied to matrices of the fifth, sixth and seventh order, confirm the validity of this approach. 
It must be noticed that the algorithms used by Mathematica $@$ are not publicly available, meaning that it was not possible to compare, from the point of view of computational complexity, the method we have used with other methods applied in the literature.

Author Contributions: Methodology, P.E.R.; software, D.C.; validation, D.C.; formal analysis, P.E.R.; investigation, P.E.R. and D.C.; data curation, D.C.; writing-original draft preparation, P.E.R.; writing-review and editing, D.C.; visualization, D.C. All authors have read and agreed to the published version of the manuscript.

Funding: This research received no external funding.

Data Availability Statement: Not applicable.

Conflicts of Interest: The authors declare no conflict of interest.

\section{References}

1. Gantmacher, F.R. The Theory of Matrices; Chelsea Pub. Co.: New York, NY, USA, 1959.

2. Higham, N.J. Functions of Matrices: Theory and Computation; SIAM: Philadelphia, PA, USA, 2008; p. xx+425.

3. Al-Mohy, A.H.; Higham, N.J. Improved inverse scaling and squaring algorithms for the matrix logarithm. SIAM J. Sci. Comput. 2012, 34, C153-C169. [CrossRef]

4. Cardoso, J.R.; Ralha, R. Matrix arithmetic-geometric mean and the computation of the logarithm. SIAM J. Matrix Anal. Appl. 2016, 37, 719-743. [CrossRef]

5. Fasi, M.; Higham, N.J. Multiprecision algorithms for computing the matrix logarithm. SIAM J. Matrix Anal. Appl. 2018, 39, 472-491. [CrossRef]

6. Miyajima, S. Verified computation for the matrix principal logarithm. Linear Algebra Appl. 2019, 569, 38-61. [CrossRef]

7. Tatsuoka, F.; Sogabe, T.; Miyatake, Y.; Zhang, S.L. Algorithms for the computation of the matrix logarithm based on the double exponential formula. J. Comput. Appl. Math. 2020, 373, 112396. [CrossRef]

8. Ibáñez, J.; Sastre, J.; Ruiz, P.; Alonso, J.M.; Defez, E. An Improved Taylor Algorithm for Computing the Matrix Logarithm. Mathematics 2021, 9, 2018. [CrossRef]

9. Hale, N.; Higham, N.J.; Trefethen, L.N. Computing $A^{\alpha}, \log (A)$, and related matrix functions by contour integrals. SIAM J. Numer. Anal. 2008, 46, 2505-2523. [CrossRef]

10. Caratelli, D.; Ricci, P.E. A numerical method for computing the roots of non-singular complex-valued matrices. Symmetry 2020, 12, 966. [CrossRef]

11. Caratelli, D.; Ricci, P.E. Inversion of tridiagonal matrices by using the Dunford-Taylor's integral. Symmetry 2021, $13,870$. [CrossRef]

12. Bruschi, M.; Ricci, P.E. An explicit formula for $f(\mathcal{A})$ and the generating function of the generalized Lucas polynomials. SIAM J. Math. Anal. 1982, 13, 162-165. [CrossRef]

13. Natalini, P.; Ricci, P.E. A "hard to die" series expansion and Lucas polynomials of the second kind. Appl. Math. 2015, 6, 1235-1240. [CrossRef]

14. Natalini, P.; Ricci, P.E. Solution of linear dynamical systems using Lucas polynomials of the second kind. Appl. Math. 2016, 7, 616-628. [CrossRef]

15. Cherubino, S. Calcolo delle Matrici; Cremonese: Roma, Italy, 1957.

16. Koch, R. Matrix Invariants. Am. Math. Mon. 1984, 91, 573-575. [CrossRef]

17. Kato, T. Perturbation Theory for Linear Operators; Springer: Berlin/Heidelberg, Germany, 1966.

18. Rudin, W. Real and Complex Analysis, 3rd ed.; Internernational Editions Mathematics Series; McGraw-Hill: Singapore, 1987.

19. Riesz, F.; Nagy, B.S. Functional Analysis; Dover: New York, NY, USA, 1990.

20. Fantappiè, L. I massimi e i minimi dei funzionali analitici reali. Atti Acc. Naz. Lincei Rend. Sci. Fis. Mat. Nat. 1930, 8, $296-301$.

21. Hirst, H.P.; Macey, W.T. Bounding the Roots of Polynomials. Coll. Math. J. 1997, 28, 292-295. [CrossRef]

22. Parodi, M. La Localisation des Valeurs Caractéristiques des Matrices et ses Applications; Gauther-Villars: Paris, France, 1959. 